\title{
STRUCTURAL BEHAVIOUR OF CROSS-LAMINATED TIMBER PANELS BY THE STATE SPACE APPROACH
}

\author{
ASAD S. ALBOSTAMI, ZHANGJIAN WU \& LEE S. CUNNINGHAM \\ School of Mechanical, Aerospace and Civil Engineering, The University of Manchester, UK.
}

\begin{abstract}
Over the last few years, Cross-Laminated Timber (CLT) panels have become increasingly popular in many structural applications. The growth in CLT uptake by the construction sector is likely to continue in line with the pressing need for sustainable construction. Although current design methods exist for CLT, often these have limits of applicability. In order to gain upmost efficiency, there is a need for improved analytical methods to fully determine the structural behaviour of CLT. In this article, CLT panels will be investigated as a novel application of the State Space Approach (SSA). As CLT is a laminated composite panel, the 3D analytical approach provided by the SSA is highly applicable. Comparison with existing experimental results for different CLT panels are explored for simply supported orthotropic CLT panels under different types of loading. The effect of the plate thickness on displacements and stresses is described quantitatively. The results demonstrate the capability of the SSA method to capture the nonlinear distribution of the stresses through the depth of the plates over a range of thicknesses, thus offering an improved understanding of CLT structural behaviour.

Keywords: analytical approach, cross-laminated timber, laminated composite panel, state space approach.
\end{abstract}

\section{INTRODUCTION}

Cross-Laminated Timber (CLT) is an engineered wood product enabling the production of large-scale load-bearing panels. The panels consist of at least three layers of boards crossstacked in alternate grain orientation and glued together on their faces, typically in a symmetric lay-up. The resulting wood product is suitable for both in-plane and out-ofplane loading, and used in structural engineering applications forming walls, floors and roofs etc.

The key advantages of CLT panels such as quick erection time and ease of handling, high strength to weight ratio and good thermal and sound insulation etc. make them highly appealing in structural engineering applications.

As a result of the increased usage of CLT panels and with a view to having a better understanding of their mechanical behaviour, new analytical approaches are needed to improve the existing design methods in terms of both accuracy and design efficiency. Towards this, several researchers have aimed at applying different analytical approaches to enhance the accuracy of the results for CLT panels. By considering the CLT as a laminated composite material, many composite plate approaches have been used including classical plate theory (CPT) and shear deformation plate theories according to Reddy [1]. Of these standard approaches, 2D theories have been most commonly investigated.

Due to the limitations of 2D plate theories, as will be explored in this article, research is ongoing to develop and apply 3D approaches to analyse and fully understand the behaviour of CLT. From a fundamental viewpoint, the accurate behaviour of the composite material should be based on 3D approaches rather than 2D approaches. However, the mathematical description of the structural behaviour of CLT in terms of accurate calculation for engineering purposes is not fully developed yet. In view of this, here we will present the SSA 
as an analytical, theoretically precise approach, which combines accuracy in defining all the displacements and the stresses in the plate with computational efficiency. A comparison of experimental tests undertaken by Hochreiner et al. [3] and existing 2D and 3D analytical methods studied by Sturzenbecher et al. [4] aims to demonstrate the potential of the SSA in capturing the behaviour of the plate for any thickness with different types of loading.

As a 3D solution, the SSA considers all displacement and transverse stress components as the primary state variables simultaneously. The boundary conditions on the top and the bottom surfaces are directly related to them. The approach originated from the Vlasov [5] state variable equation for the solution of 3D elasticity problems by using the method of initial functions. Later, it was developed by Bahar [6] as the state space approach for homogeneous and isotropic plates. As one of the pioneering researchers in 3D elastic theory, $\mathrm{Wu}$ [7] introduced SSA to a 3D thick plate analysis. Fan and Ye [8] presented a theoretically exact solution based on the state space method for statics and dynamics of orthotropic thick plates with simply supported edges. All fundamental equations of three-dimensional elasticity can be exactly satisfied and the nine elastic constants for orthotropic materials can be considered. Besides that, the approach extended by Fan and Ye can be applied to the buckling of a thick orthotropic plate. Likewise, Wu and Wardenier [9] achieved an exact 3D elasticity solution for simply supported thick, orthotropic and rectangular plates subjected to arbitrary loading. In fact, they obtained a sixth-order differential equation governing the transverse displacement for the first time in comparison with the fourth-order differential equation that is used in CPT.

\subsection{Experimental and theoretical investigations for CLT}

To understand CLT itself, a significant amount of experimental investigations have been undertaken over the last 10 to 15 years. To gain an insight into the accuracy of current design approaches, particularly for bending, Hochreiner et al. [3] focused on the global failure mechanisms and the different rupture patterns in three- and five-layered CLT plates. Czaderski et al. [10] investigated the mechanical behaviour of three-layered CLT plates with different thickness to width ratios from 0.025 To 0.25 under three different types of loadings. The aim of the tests was to estimate the actual modulus of elasticity and the strength of the bonded panels. They also compared their data with existing beam and plate methods. Similarly, a detailed plate-bending experiment on CLT panels was carried out by O'Dowd et al. [11]. For their experiment, they measured the accurate bending stiffness for three different sample sizes of a five laminae CLT panel with samples from each lamina being subject to three separate bending tests. In addition, three different theoretical methods (Gamma-method, $K$-method and shear analogy method [12]) were compared with the bending stiffness obtained experimentally. O'Dowd et al. [11] found that the Gamma method gives reliably conservative results while the $K$-method and shear analogy method underestimate the experimental stiffness. In another experiment investigated by Sikora et al. [13] the effect of the thickness of the CLT panels on the bending and shear strength was studied using the same three theoretical methods investigated by [11]. It was found that the bending strength decreased as the thickness of the CLT panel increased, and similar effects were observed for the rolling shear strength. It should be noted that [13] considered only thin plates. In addition, it was also noticed that the type of failure was determined by the bonding process used to form the CLT panel and the resulting quality of the bond. 
From the theoretical viewpoint, Sturzenbecher and Hofstetter [2] and Sturzenbecher et al. [4] applied 2D and quasi-3D approaches to several CLT laminate lay-ups of three to seven layers and different thickness to width ratios from 0.25 to 0.025 under various transverse loading conditions, comparing the accuracy of each. 2D theories such as CPT, First order Shear Deformation Theory (FSDT) and more advanced theories as outlined in Ren [14] were applied. The results from the 2D theories were compared with the quasi-3D elasticity solutions given by Pagano [15] and a good agreement was obtained. However, the focussed 2D approaches had limitations: for example, the effect of the transverse normal stress $\left(\sigma_{z}\right)$ is neglected and the deflection $(w)$ is assumed constant across the thickness of the plate. Although in [2, 4], the Pagano solution was defined as an exact solution, it is still considered as a quasi-3D solution as it did not include the normal transverse stress in the equilibrium equation. Note here that the term 'exact solution' refers to a solution that captures the entire physics of the problem within the context of an idealised, perfectly elastic material [16]. In addition, they compared the various analytical results with experimental data by Czaderski et al. [10], and they found that the 2D theories fail to get accurate solutions in comparison to the numerical and experimental results for thick CLT plates with a thickness to width ratio of 0.2 and more, as well as for concentrated loads.

Developing more advanced theoretical solutions for the analysis of CLT panels is an active research topic due to the complicated behaviour of the CLT elements. More accurate theories have the potential to provide a better understanding of CLT panel structural performance and enable their efficient and safe application in construction. The following sections introduce the SSA method considering it as a theoretically ideal solution and its application to CLT is explored via two case studies from refs. [3,4] comparing the experimental test results and the different approaches described by these two references.

\section{SSA GOVERNING EQUATION}

\subsection{Formulation of the equation}

Consider an elastic homogeneous orthotropic rectangular plate of length $a$, width $b$ and uniform thickness $h$ as shown in Fig. 1, the elasto-static equilibrium equations of the plate [17] can be written as

$$
\frac{\partial \sigma_{x}}{\partial x}+\frac{\partial \tau_{x y}}{\partial y}+\frac{\partial \tau_{x z}}{\partial z}=0 ; \frac{\partial \tau_{x y}}{\partial x}+\frac{\partial \sigma_{y}}{\partial y}+\frac{\partial \tau_{y z}}{\partial z}=0 ; \frac{\partial \tau_{x z}}{\partial x}+\frac{\partial \tau_{y z}}{\partial y}+\frac{\partial \sigma_{z}}{\partial z}=0
$$

Where $\sigma_{x}, \sigma_{y}$ and $\sigma_{z}$ are normal stresses along $x, y$ and $z$ axes, and $\tau_{x y}, \tau_{y z}$ and $\tau_{x z}$ are shear stresses with respect to $x-y, y-z$ and $x-z$ planes respectively.

Since Hook's law can be applied to elastic materials, the stress-strain relation becomes

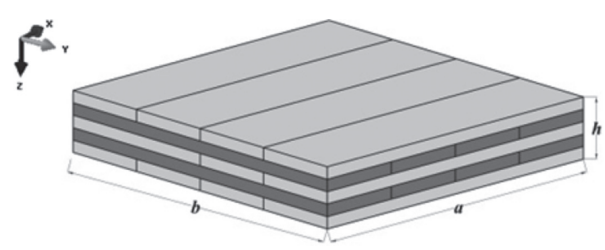

Figure 1: Geometry of a plate. 


$$
\left\{\begin{array}{c}
\sigma_{x} \\
\sigma_{y} \\
\sigma_{z} \\
\tau_{y z} \\
\tau_{x z} \\
\tau_{x y}
\end{array}\right\}=\left[\begin{array}{cccccc}
C_{11} & C_{12} & C_{13} & 0 & 0 & 0 \\
C_{21} & C_{22} & C_{23} & 0 & 0 & 0 \\
C_{31} & C_{32} & C_{33} & 0 & 0 & 0 \\
0 & 0 & 0 & C_{44} & 0 & 0 \\
0 & 0 & 0 & 0 & C_{55} & 0 \\
0 & 0 & 0 & 0 & 0 & C_{66}
\end{array}\right]\left\{\begin{array}{c}
\varepsilon_{x} \\
\varepsilon_{y} \\
\varepsilon_{z} \\
\gamma_{y z} \\
\gamma_{x z} \\
\gamma_{x y}
\end{array}\right\}
$$

In eqn (2), the strain-displacement relations can be written as

$$
\varepsilon_{x}=\frac{\partial u}{\partial x}, \varepsilon_{y}=\frac{\partial v}{\partial y}, \varepsilon_{z}=\frac{\partial w}{\partial z}, \gamma_{y z}=\frac{\partial v}{\partial z}+\frac{\partial w}{\partial y}, \gamma_{x z}=\frac{\partial u}{\partial z}+\frac{\partial w}{\partial x}, \gamma_{x y}=\frac{\partial u}{\partial y}+\frac{\partial v}{\partial x}
$$

and

$$
\begin{aligned}
C_{11} & =\frac{E_{1}\left(1-v_{23} v_{32}\right)}{Q} C_{12}=\frac{E_{1}\left(v_{21}+v_{31} v_{23}\right)}{Q} C_{22}=\frac{E_{2}\left(1-v_{13} v_{31}\right)}{Q} \\
C_{13} & =\frac{E_{1}\left(v_{31}+v_{21} v_{32}\right)}{Q} C_{33}=\frac{E_{3}\left(1-v_{12} v_{21}\right)}{Q} C_{23}=\frac{E_{2}\left(v_{32}+v_{12} v_{31}\right)}{Q} \\
C_{44} & =G_{23} \quad C_{55}=G_{31} \quad C_{66}=G_{12} \\
Q & =1-v_{12} v_{21}-v_{23} v_{32}-v_{31} v_{13}-2 v_{12} v_{23} v_{31} \\
\frac{v_{i j}}{E_{i}}= & \frac{v_{j i}}{E_{j}}(i, j=1,2,3)
\end{aligned}
$$

Where $E_{1}, E_{2}$ and $E_{3}$ are the Young's moduli of the plate among the material coordinates.

The subscripts 1, 2 and 3 indicate fibre direction, transverse and perpendicular to the plate respectively. $G_{12}, G_{23}$ and $G_{13}$ are shear moduli with respect to 1-2, 2-3 and 1-3 planes respectively. $v_{12}, v_{23}$ and $v_{13}$ are the Poisson's ratios correspondingly.

From eqn (2), all stress components can be expressed explicitly:

$$
\begin{gathered}
\sigma x=C_{11} \frac{\partial u}{\partial x}+C_{12} \frac{\partial v}{\partial y}+C_{13} \frac{\partial w}{\partial z} ; \sigma y=C_{12} \frac{\partial u}{\partial x}+C_{22} \frac{\partial v}{\partial y}+C_{23} \frac{\partial w}{\partial z} \\
\sigma z=C_{13} \frac{\partial u}{\partial x}+C_{23} \frac{\partial v}{\partial y}+C_{33} \frac{\partial w}{\partial z} \\
\tau_{y z}=C_{44}\left(\frac{\partial v}{\partial z}+\frac{\partial w}{\partial y}\right) ; \tau_{x z}=C_{55}\left(\frac{\partial u}{\partial z}+\frac{\partial w}{\partial x}\right) ; \tau_{x y}=C_{66}\left(\frac{\partial u}{\partial y}+\frac{\partial v}{\partial x}\right)
\end{gathered}
$$

Three displacements in $x-y-z$ directions, labelled with $u, v$ and $w$, respectively, and the transverse stresses $\sigma_{z}, \sigma_{x z}$ and $\sigma_{y z}$, are solved with respect to the $z$ coordinate directly from eqns (1), (3) and (5), 
Denoting

$$
\begin{aligned}
& C_{1}=-\frac{C_{12}}{C_{33}} C_{2}=C_{11}-\frac{C_{12}^{2}}{C_{33}} C_{3}=C_{12}-\frac{C_{13} C_{23}}{C_{33}} \\
& C_{4}=C_{22}-\frac{C_{23}^{2}}{C_{33}} C_{5}=-\frac{C_{23}}{C_{33}} C_{6}=C_{66} \\
& C_{7}=\frac{1}{C_{33}} C_{8}=\frac{1}{C_{55}} C_{9}=\frac{1}{C_{44}}
\end{aligned}
$$

and rearranging eqn (5) leads to a matrix form as:

$$
\frac{\partial}{\partial z}\{F\}=[G]\{F\}
$$

Herein, $\{\mathrm{F}\}=\left[u v \sigma_{z} \tau_{x z} \tau_{y z} w\right]^{T}$ is called the state vector of the plate and [G] is named as the system state matrix

$$
[\mathrm{G}]=\left[\begin{array}{cccccc}
0 & 0 & 0 & C_{8} & 0 & -\frac{\partial}{\partial x} \\
0 & 0 & 0 & 0 & C_{9} & -\frac{\partial}{\partial y} \\
0 & 0 & 0 & -\frac{\partial}{\partial x} & -\frac{\partial}{\partial y} & 0 \\
-C_{2} \frac{\partial^{2}}{\partial x^{2}}-C_{6} \frac{\partial^{2}}{\partial y^{2}} & -\left(C_{3}+C_{6}\right) \frac{\partial^{2}}{\partial x \partial y} & C_{1} \frac{\partial}{\partial x} & 0 & 0 & 0 \\
-\left(C_{3}+C_{6}\right) \frac{\partial^{2}}{\partial x \partial y} & -C_{6} \frac{\partial^{2}}{\partial x^{2}}-C_{4} \frac{\partial^{2}}{\partial y^{2}} & C_{5} \frac{\partial}{\partial y} & 0 & 0 & 0 \\
C_{1} \frac{\partial}{\partial x} & C_{5} \frac{\partial}{\partial y} & C_{7} & 0 & 0 & 0
\end{array}\right]
$$

Once the state vector containing the three displacements and the transverse stresses $\left[\sigma_{z}, \tau_{x z}, \tau_{y z}\right]$ has been found, the three in-plane stresses in the $x-y$ plane can be calculated from eqn (5) as follows:

$$
\left\{\begin{array}{l}
\sigma_{x} \\
\sigma_{y} \\
\tau_{x y}
\end{array}\right\}=\left[\begin{array}{cccccc}
C_{2} \frac{\partial}{\partial x} & C_{3} \frac{\partial}{\partial y} & -C_{1} & 0 & 0 & 0 \\
C_{3} \frac{\partial}{\partial x} & C_{4} \frac{\partial}{\partial y} & -C_{5} & 0 & 0 & 0 \\
C_{6} \frac{\partial}{\partial y} & C_{6} \frac{\partial}{\partial x} & 0 & 0 & 0 & 0
\end{array}\right]\left\{\begin{array}{c}
u \\
v \\
\sigma_{z} \\
\tau_{x z} \\
\tau_{y z} \\
w
\end{array}\right\}
$$

To consider a rectangular plate with the boundary conditions for the four sides of the simply supported plate (Fig. 1), the following boundary condition should be satisfied: 


$$
\sigma_{x}=v=w=0(\text { at } x=0, a) \text { and } \sigma_{y}=u=w=0(\text { at } y=0, b)
$$

In order to satisfy the boundary conditions specified in eqn (10), the following six state variables of the state vector can be expressed by:

$$
\begin{aligned}
u(x, y, z) & =\sum_{m=1} \sum_{n=1} U_{m n}(z) \cos (m \pi x / a) \sin (n \pi y / b) \\
v(x, y, z) & =\sum_{m=1} \sum_{n=1} V_{m n}(z) \sin (m \pi x / a) \cos (n \pi y / b) \\
w(x, y, z) & =\sum_{m=1} \sum_{n=1} W_{m n}(z) \sin (m \pi x / a) \sin (n \pi y / b) \\
\tau_{x z}(x, y, z) & =\sum_{m=1} \sum_{n=1} X_{m n}(z) \cos (m \pi x / a) \sin (n \pi y / b) \\
\tau_{y z}(x, y, z) & =\sum_{m=1} \sum_{n=1} Y_{m n}(z) \sin (m \pi x / a) \cos (n \pi y / b) \\
\sigma_{z}(x, y, z) & =\sum_{m=1} \sum_{n=1} Z_{m n}(z) \sin (m \pi x / a) \sin (n \pi y / b)
\end{aligned}
$$

Let $\zeta=m \pi / a$ and $\eta=n \pi / b$ where $m$ and $n$ are the number of loops of the analytical solution in the $x$ and $y$ directions respectively and substituting eqn (11) into eqn (7) yields for each combination of $m$ and $n$

$$
\frac{\partial}{\partial z}\left\{\mathrm{~F}_{m n}(z)\right\}=\left[\mathrm{G}_{m n}\right]\left\{\mathrm{F}_{m n}(z)\right\}
$$

Herein, $\left\{\mathrm{F}_{m n}(z)\right\}=\left[U_{m n}(z) V_{m n}(z) Z_{m n}(z) X_{m n}(z) Y_{m n}(z) W_{m n}(z)\right]^{T}$ is the state vector of the plate with the number of looping $m-n^{\text {th }}$ and the system matrix is

$$
\left[\mathrm{G}_{m n}\right]=\left[\begin{array}{cccccc}
0 & 0 & 0 & \mathrm{C}_{8} & 0 & -\zeta \\
0 & 0 & 0 & 0 & \mathrm{C}_{9} & -\eta \\
0 & 0 & 0 & \zeta & \eta & 0 \\
\mathrm{C}_{2} \zeta^{2}+\mathrm{C}_{6} \eta^{2} & \left(\mathrm{C}_{3}+\mathrm{C}_{6}\right) \zeta \eta & \mathrm{C}_{1} \zeta & 0 & 0 & 0 \\
\left(\mathrm{C}_{3}+\mathrm{C}_{6}\right) \zeta \eta & \mathrm{C}_{6} \zeta^{2}+\mathrm{C}_{4} \eta^{2} & \mathrm{C}_{5} \eta & 0 & 0 & 0 \\
-\mathrm{C}_{1} \zeta & -\mathrm{C}_{5} \eta & \mathrm{C}_{7} & 0 & 0 & 0
\end{array}\right]
$$

2.2 Solution of the equation

By solving the differential eqn (12) based on classical solution method of a linear differential equation [18], the solution can be found as:

$$
\left\{\mathrm{F}_{m n}(z)\right\}=\left[\mathrm{D}_{m n}(z)\right]\left\{\mathrm{F}_{m n}(0)\right\}
$$


where $\left[\mathrm{D}_{m n}(z)\right]=\exp \left\{\left[\mathrm{G}_{m n}\right] z\right\}$, and when $z=h$,

$$
\left\{\mathrm{F}_{m n}(h)\right\}=\left[\mathrm{D}_{m n}(h)\right]\left\{\mathrm{F}_{m n}(0)\right\}
$$

$\left\{\mathrm{F}_{m n}(0)\right\}$ and $\left\{\mathrm{F}_{m n}(h)\right\}$ are the values of the state variables on top $(z=0)$ and bottom $(z=h)$ surfaces. They can be determined uniquely based on the load conditions on the top and bottom surfaces of the plate. For example, if the plate is subjected to an arbitrarily distributed external transverse pressure $q(x, y)$ on the top surface only, the state vectors on the top and bottom surfaces of the plate can be expanded into a Fourier series as shown in eqns (16) and (17), respectively:

$$
\left\{\mathrm{F}_{m n}(0)\right\}=\left\{\begin{array}{c}
U_{m n}(0) \\
V_{m n}(0) \\
\frac{4}{a b} \int_{0}^{a b} q(x, y) \sin (m \pi x / a) \sin (n \pi y / b) d x d y \\
0 \\
0 \\
W_{m n}(0)
\end{array}\right\}
$$

and

$$
\left\{\mathrm{F}_{m n}(h)\right\}^{T}=\left[U_{m n}(h) V_{m n}(h) 000 W_{m n}(h)\right]
$$

Substituting eqns (16) and (17) into (15), it is easy to get all displacement components on the top and the bottom surfaces of the plate, that is $U_{m n}(0), V_{m n}(0), W_{m n}(0)$ and $U_{m n}(h), V_{m n}(h), W_{m n}(h)$.

After finding all the displacements and the transverse stresses, the in-plane stresses can be found by using the following equation:

$$
\left\{\begin{array}{c}
\sigma_{x} \\
\sigma_{y} \\
\tau_{x y}
\end{array}\right\}=\left[\begin{array}{cccccc}
-C_{2} \zeta & -C_{3} \eta & -C_{1} & 0 & 0 & 0 \\
-C_{3} \zeta & -C_{4} \eta & -C_{5} & 0 & 0 & 0 \\
C_{6} \eta & C_{6} \zeta & 0 & 0 & 0 & 0
\end{array}\right]\left\{\begin{array}{c}
u \\
v \\
\sigma_{z} \\
\tau_{x z} \\
\tau_{y z} \\
w
\end{array}\right\}
$$

Further formulation simplifications can be used if $q(x, y)=q$ is a constant. The SSA method has previously been applied to a generic composite plate by the authors [19] and the same basic geometry (Fig. 1) shall be used henceforth. For laminated plates as shown in Fig. 2, eqn (15) will be extended to:

$$
\left\{F_{m n}\left(d_{j}\right)\right\}_{j}=\left[G\left(d_{j}\right)\right]_{j}\left\{F_{m n}(0)\right\}_{j}
$$

and for $(j+1)^{\text {th }}$ layer;

$$
\left\{F_{m n}\left(d_{j+1}\right)\right\}_{j+1}=\left[G\left(d_{j+1}\right)\right]_{j+1}\left\{F_{m n}(0)\right\}_{j+1}
$$




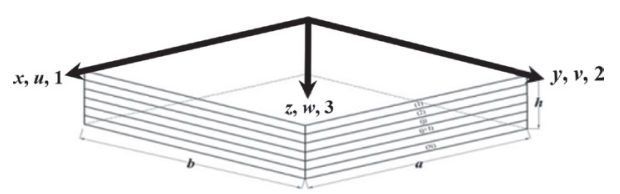

Figure 2: Nomenclature of an orthotropic rectangular laminated plate.

Therefore,

$$
\left\{F_{m n}\left(d_{j+1}\right)\right\}_{j+1}=\left[G\left(d_{j+1}\right)\right]_{j+1}\left[G\left(d_{j}\right)\right]_{j}\left\{F_{m n}(0)\right\}_{j}
$$

Due to $\left\{F_{m n}(0)\right\}_{j+1}=\left\{F_{m n}\left(d_{j}\right)\right\}_{j}$, eqn (19) can be summarised as the following equation:

$$
\left\{F m n\left(d_{j+1}\right)\right\}_{j+1}=[\Pi]\{F m n(0)\}_{j+1}
$$

Where;

$$
\Pi=\left[G\left(d_{j+1}\right)\right]_{j+1}\left[G\left(d_{j}\right)\right]_{j}
$$

\section{APPLICATION TO CLT}

In the following section, two case studies with CLT panels under different types of loadings will be analysed by use of the SSA. For these cases, the CLT panels are considered as an orthotropic material with three principal axes, longitudinal axis parallel to the grain, radial and tangential axes. The last two axes are treated to be the same in value and regarded as having properties equivalent to those perpendicular to the grain [20]. Also, the boundary conditions are simply supported on all sides.

\subsection{Case study (1)}

For this case study, the panel has the same geometry and properties as the experiment tested by Hochreiner et al. [3]. The panel is symmetrical with five layers of alternate grain orientation i.e. $[0,90,0,90,0]^{\circ}$ under central concentrated loading acted uniformly on a patch area $(c, d)$ as shown in Fig. 3. Each layer has a thickness of $19 \mathrm{~mm}$, so the total plate thickness is

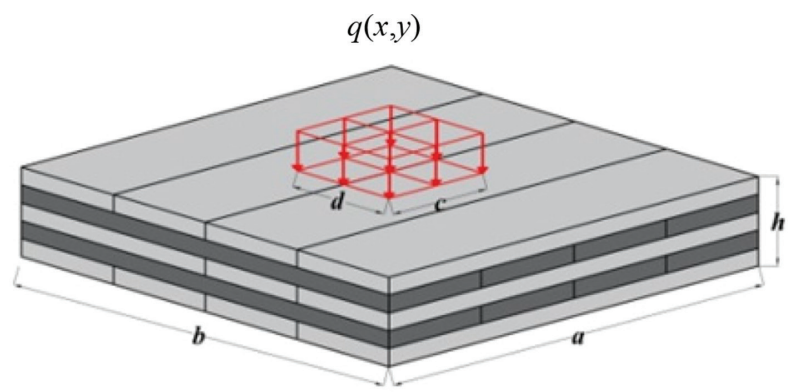

Figure 3: Geometry of a panel under central concentrated loading. 
$95 \mathrm{~mm}$. The in-plane dimensions of the plate are $a=b$ and $h / a$ is equal to 0.05 . Here, $a$ and $b$ are the length of the plate along the $x$ axis and $y$ axis, respectively and $h$ is the total thickness of the plate (z-axis) as shown in Fig. 3. The set of elastic material parameters used for the timber layers are as the following and as per reference [3]: $E_{1}=11000 \mathrm{MPa}, E_{2}=370 \mathrm{MPa}$, $E_{3}=370 \mathrm{MPa}, G_{12}=690 \mathrm{MPa}, G_{13}=690 \mathrm{MPa}, G_{23}=50 \mathrm{MPa}, \mu_{12}=0.44, \mu_{13}=0.44$ and $\mu_{23}=0.64$.

As shown from Fig. 4a, the SSA gives very good agreement with experimental results for the out-of-plane displacement $(w)$. From this point of view, the SSA can be adopted for this case study and the out-of-plane displacement $(w)$ can be drawn through the thickness of the CLT plate as shown from Fig. 4b. From this figure, the nonlinear behaviour of $(w)$ is clearly noticed.

As $h / a$ is equal to 0.05 , the CLT plate is considered as a thin plate; however, by applying the SSA for this case study, the actual behaviour of the in-plane stresses $\left(\sigma_{x}\right)$ through the thickness can be noticed as shown in Fig. 5 and the application can be extended to thicker plates where the 2D methods are unable to capture the stresses accurately. Also, as shown in the same figure, the in-plane stresses $\left(\sigma_{x}\right)$ decreased as the thickness of the plate is increased. In addition, in the first layer as the thickness is increased the stresses will change sign from negative to positive.

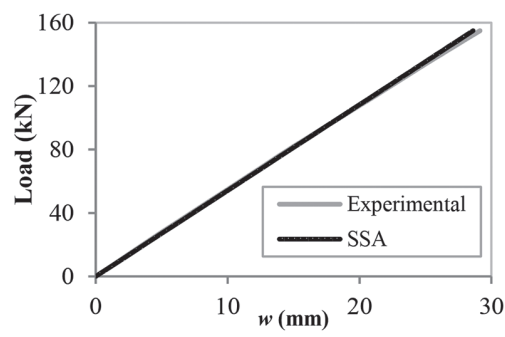

(a)

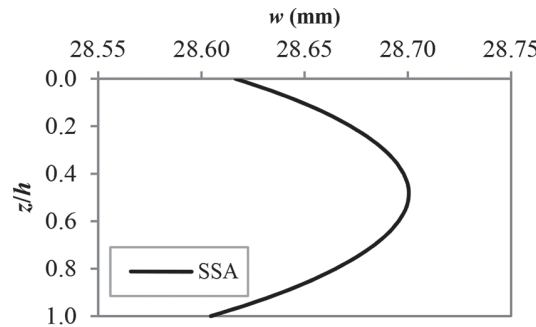

(b)

Figure 4: (a) Load-displacement curves for the experimental and SSA and (b) SSA out -ofplane displacement $(w)$ distribution through the thickness.

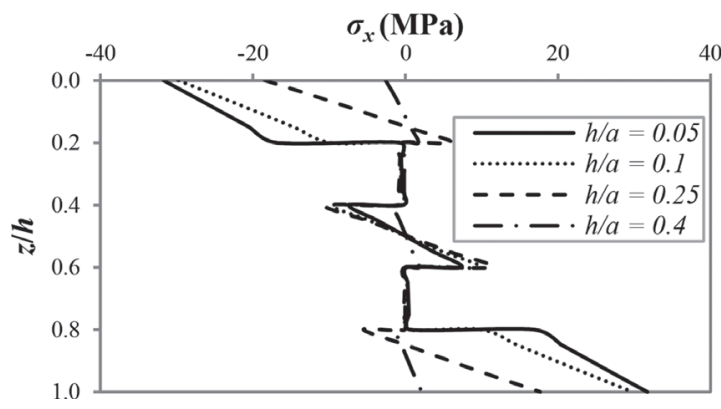

Figure 5: The effect of different $h / a$ on the in-plane stress $\left(\sigma_{x}\right)$ through the thickness for case study (1). 


\subsection{Case study (2)}

For this case study, the panel has the same geometry as that examined analytically by Sturzenbecher et al. [4] The panel is symmetrical with five layers as in case study (1) under a bi-sinusoidal load with a maximum magnitude of $0.5 \mathrm{MPa}$ as shown in Fig. 6. Each layer has a thickness of $30 \mathrm{~mm}$, so that the total plate thickness is $150 \mathrm{~mm}$. The in-plane dimensions of the plate are $a=b$ and $h / a$ is equal to 0.1 . The set of elastic material parameters used for the timber layers are as case study (1). All the calculations are based on C24-grade timber [21] as the constituent of CLT boards, with properties in accordance with BS EN $338[22]$.

In Fig. 7, the SSA is compared with CPT and Pagano solutions for the in-plane and out-ofplane displacement $(u$ and $w$ ). For both scenarios, the SSA gives a very good agreement with Pagano results. CPT gives a linear distribution through the thickness as shown in Fig. 7a. From Fig. 7b, it can be seen that while the SSA and Pagano results have good agreement, there is a large difference with the CPT results, thus highlighting the limitations of the CPT method.

In this case study, $h / a$ is equal to 0.1 , thus the plate is considered as a thin CLT plate. As the CLT becomes thicker, the SSA is capable of accurately capturing all the elastic stresses and the displacement through the thickness as shown from Figs 8 and 9.

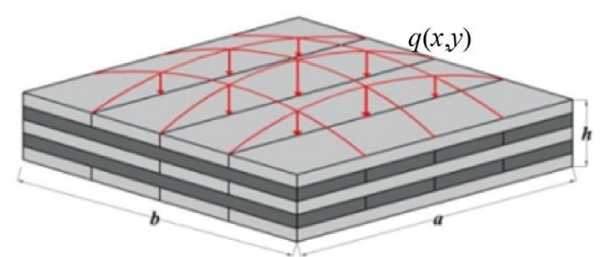

Figure 6: Geometry of a panel under bi-sinusoidal loading.

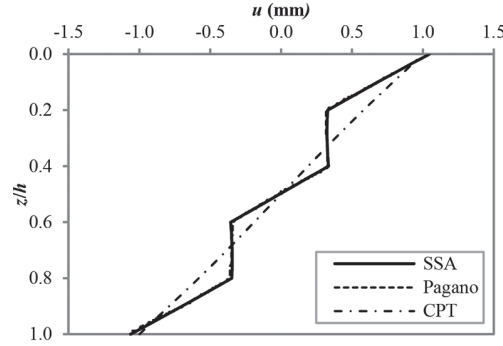

(a)

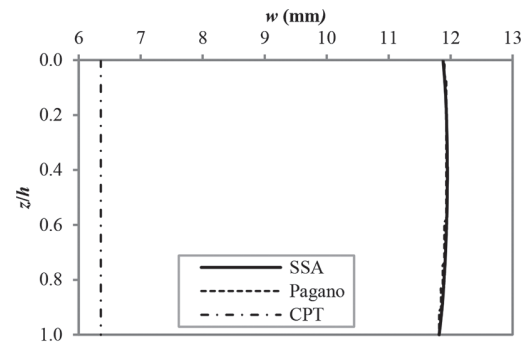

(b)

Figure 7: Displacement distribution through the thickness of CLT-5 ply plate for: (a) in-plane $u$ and (b) out-of-plane $w$ displacements. 


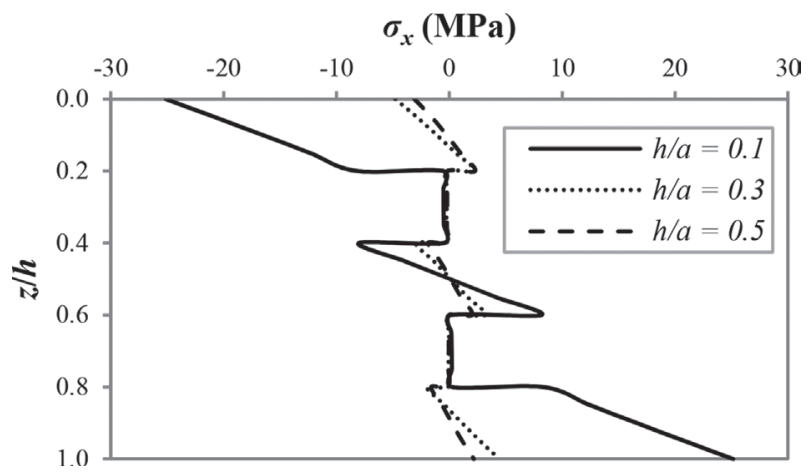

Figure 8: The effect of different $h / a$ on the in-plane stress $\left(\sigma_{x}\right)$ through the thickness for case study (2).
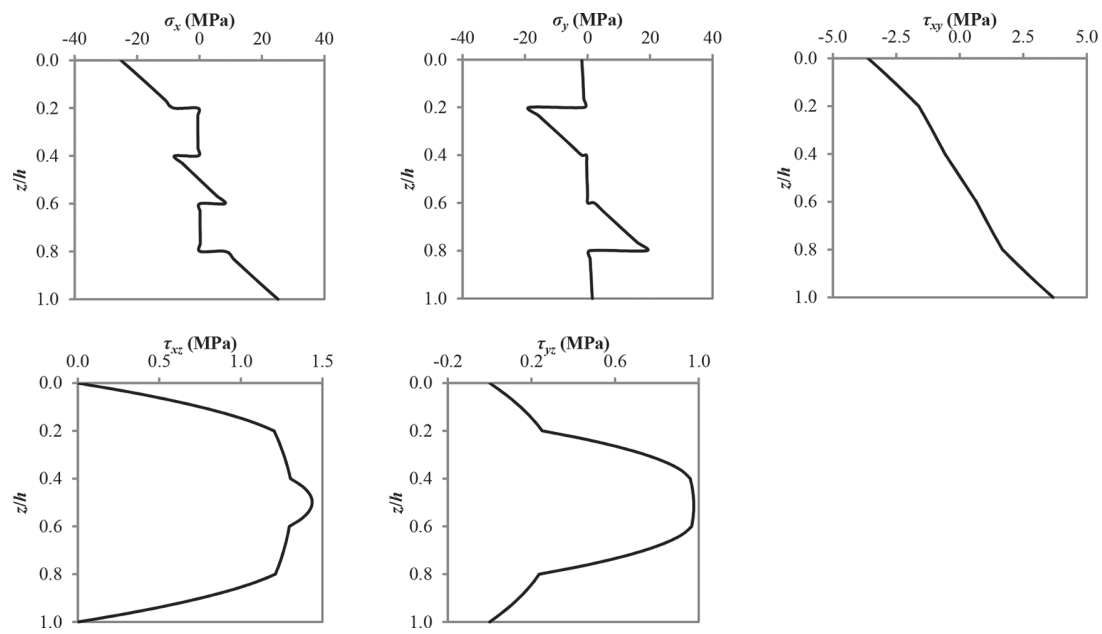

Figure 9: In-plane and out-of-plane displacements and stresses states of CLT-5 ply plate for $h / a=0.1$ by SSA.

\section{CONCLUSION}

The SSA has the potential to improve accuracy and range of applicability over commonly used methods as the existing analytical approaches have limitations in applicability and accuracy. Towards that, an analytical investigation has been carried out for a simply supported orthotropic CLT panel under two types of loading, a central concentrated loading and a bisinusoidal load. A comparison between the SSA, experimental test and various existing analytical approaches has been carried out. The following conclusions can be drawn:

- By using the SSA, thicker CLT panels can be modelled in both case studies and all the stresses and the displacements can be analysed at any location along the $x$ and $y$ directions and through the thickness.

- As the method used by Sturzenbecher et al. [4] neglects $\sigma_{z}$ and $w$ is assumed constant through the thickness of the plate, by applying the SSA to the CLT panels, the transverse 
normal stress $\sigma_{z}$ is included in the calculations and the out-of-plane displacement $w$ can be calculated exactly at any location through the thickness. Also, the nonlinear behaviour of $w$ is clearly noticed by using the SSA.

- The SSA gives a very good agreement with the experimental test result in the first case study and a good agreement with the Pagano method in the second case study.

\section{ACKNOWLEDGEMENT}

The authors gratefully acknowledge the contribution of the University of Petra, Jordan, to funding this research.

\section{REFERENCES}

[1] Reddy, J.N., Mechanics of Laminated Composite Plates and Shells: Theory and Analysis. CRC press, 2004.

[2] Sturzenbecher, R. \& Hofstetter, K., Bending of cross-ply laminated composites: an accurate and efficient plate theory based upon models of lekhnitskii and ren. Composite Structures, 93(3), pp. 1078-1088, 2011.

https://doi.org/10.1016/j.compstruct.2010.09.020

[3] Hochreiner, G., Fussl, J. \& Eberhardsteiner, J., Cross-laminated timber plates subjected to concentrated loading: experimental identification of failure mechanisms. Strain, 50(1), pp. 68-81, 2014. https://doi.org/10.1111/str.12068

[4] Sturzenbecher, R., Hofstetter, K. \& Eberhardsteiner, J., Structural design of cross laminated timber (CLT) by advanced plate theories. Composites Science and Technology, 70(9), pp. 1368-1379, 2010.

https://doi.org/10.1016/j.compscitech.2010.04.016

[5] Vlasov, V., Method of initial functions in problems of theory of thick plates and shells. Proceedings of the ninth of the International Congresses on Theoretical and Applied Mechanics. Brussels, p. 321, 1957.

[6] Bahar, LY., A state space approach to elasticity. Journal of Franklin Institute, pp. 33-41, 1975. https://doi.org/10.1016/0016-0032(75)90082-4

[7] Wu, Z.J., Exact solution of orthotropic simply-supported rectangular plates under arbitrary loadings. University of Hefei Technology: Hefei, China, 1987.

[8] Fan, J. \& Ye, J., An exact solution for the statics and dynamics of laminated thick plates with orthotropic layers. International Journal of Solids and Structures, 26(7), pp. 655662, 1990.

[9] Wu, ZJ. \& Wardenier, J., Further investigation on the exact elasticity solution for anisotropic thick rectangular plates. International Journal of Solids and Structures, 35(7-8), pp. 747-758, 1998.

https://doi.org/10.1016/S0020-7683(97)00070-X

[10] Czaderski, C., Steiger, R., Howald, M., Olia, S., Gülzow, A. \& Niemz, P., Versuche und Berechnungen an Allseitig Gelagerten 3-Schichtigen Brettsperrholzplatten. Holz als Roh - und Werkst, 65(5), pp. 383-402, 2007. https://doi.org/10.1007/s00107-007-0184-6

[11] O’Dowd, B., Cunningham, L.S. \& Nedwell, P., Briefing : Experimental and theoretical bending stiffness of cross-laminated timber panels. Proceedings of the Institution of Civil Engineers - Construction Materials, 169(6), pp. 277-281, 2016.

https://doi.org/10.1680/jcoma.15.00063 
[12] FPInnovations. CLT Handbook Canadian Edition. Journal of Chemical Information and Modeling, 53, pp. 1689-1699, 2013.

[13] Sikora, K.S., McPolin, D.O. \& Harte, A.M., Effects of the thickness of cross-laminated timber (CLT) panels made from irish sitka spruce on mechanical performance in bending and shear. Construction and Building Materials, 116, pp. 141-150, 2016. https://doi.org/10.1016/j.conbuildmat.2016.04.145

[14] Ren, J.G., Bending theory of laminated plate. Composites Science and Technology, 27(3), pp. 225-248, 1986. https://doi.org/10.1016/0266-3538(86)90033-3

[15] Pagano, N.J., Exact solutions for composite laminates in cylindrical bending. Composite Materials, 3, pp. 398-411, 1987. https://doi.org/10.1177/002199836900300304

[16] Mathematica Tutorial. 2016.

[17] Ye, J., Laminated Composite Plates and Shells, London: Springer London, 2003. https://doi.org/10.1007/978-1-4471-0095-9

[18] Stauder, C., Cross-Laminated Timber: An Analysis of the Austrian Industry and Ideas for Fostering its Development in America, Austria, 2013.

[19] Albostami, A., Wu, Z. \& Zou, Z., Precise determination of structural behaviour of composite plates. Proceeding of MACE PGR Conference, Manchester, pp. 21-23, 2015.

[20] Mckenzie, Design of Structural Timber, London: Macmillan Press LTD, 2000.

[21] BS EN 408:2010+A1, Timber structures - structural timber and glued laminated timber - determination of some physical and mechanical properties, BSI, Milton Keynes, UK, 2012.

[22] BS EN 338, Structural timber - strength classes, BSI, Milton Keynes, UK, 2009. 